\title{
Open Spin Chains in Super Yang-Mills at Higher Loops: Some Potential Problems with Integrability
}

\author{
Abhishek Agarwal
}

\author{
Physics Department \\ City College of the CUNY \\ New York, NY 10031 \\ abhishek@sci.ccny.cuny.edu
}

\begin{abstract}
The super Yang-Mills duals of open strings attached to maximal giant gravitons are studied in perturbation theory. It is shown that non-BPS baryonic excitations of the gauge theory can be studied within the paradigm of open quantum spin chains even beyond the leading order in perturbation theory. The open spin chain describing the two loop mixing of non-BPS giant gravitons charged under an $s u(2)$ of the so(6) $R$ symmetry group is explicitly constructed. It is also shown that although the corresponding open spin chain is integrable at the one loop order, there is a potential breakdown of integrability at two and higher loops. The study of integrability is performed using coordinate Bethe ansatz techniques.
\end{abstract}




\section{Introduction and Summary}

In the present paper we study the mixing of non-BPS giant gravitons in $\mathcal{N}=4 \mathrm{SYM}$ beyond the leading order in the 't Hooft coupling. We pay special attention to open-string like operators coupled to maximal giant gravitons built out of two of the three complex scalars $Z, W$ at two loop order. We show by an explicit computation that the two loop mixing of such operators can be described within the paradigm of open spin chains and we construct the two loop open spin chain. We thus extend the work of Berenstein and Vazquez [4, 5] who had shown that the one loop mixing of non-BPS giant gravitons can be described by integrable open spin chains. Our analysis suggests that although the spin chain paragigm holds beyond the leading order in perturbation theory, the two loop open spin chain is not integrable.

The discovery of integrability in $\mathcal{N}=4 \mathrm{SYM}[1$, has lead to various remarkable insights in the present day understanding of $\mathcal{N}=4 \mathrm{SYM}$ and many of its less and non-supersymmetric cousins. The fact that in 't Hooft's large $N$ limit the mixing of single trace operators of the gauge theory can be studied and the spectrum of anomalous dimensions of the gauge theory computed by diagonalizing an associated one dimensional quantum spin chain, which, to the extent that it has been possible to check by explicit computations, appears to be integrable opens up the possibility of detailed higher loop studies of the gauge theory. Integrability in superconformal Yang-Mills theory was first discovered at the one loop level in the seminal paper of Minahan and Zarembo[1] who showed that the mixing of single trace operators constructed out of the six (real) scalars of the gauge theory is described by an integrable $S O(6)$ spin chain. This result has subsequently been generalized and supersymmetrized to incorporate the mixing of single trace operators involving any of the SYM fields at the one loop level[2]. At this level, the mixing is described by a supersymmetric closed spin chain, which nonetheless is integrable.

The status of integrability, beyond the one loop order is also very promising. Higher loop integrability in the gauge theory, which was first glimpsed in 3 . has since been understood in much grater detail. It was shown by Staudacher 8 that higher loop integrability; at least in some closed sub-sectors of operator mixing such as the su(2) sector built out of two complex scalars, the $s l(2)$ sector involving a complex scalar and a single covariant derivative and the $s u(1 \mid 1)$ sector involving a complex scalar and a fermion, can be made manifest

as one can write down a set of Bethe equations whose solutions determine the spectra of anomalous dimensions of the gauge theory and also produce the factorized scattering matrix of the closed spin chains that describe these sectors. The insights gained from the Bethe equations that determine the spectra in these various closed 'two-component' sectors have been utilized by Beisert and Staudacher in [6] where a remarkable all loop Bethe ansatz was proposed. Assuming the correctness of this Bethe ansatz, one is lead to the extremely 
pleasant situation where the all-loop large $N$ spectrum of anomalous dimensions of all single trace operators of $N=4 \mathrm{SYM}$ is described by an underlying integrable quantum spin chain. Though, one does not yet know the Hamiltonian of this complete supersymmetric spin chain one can write down the all loop Hamiltonian in some less supersymmetric sectors. For example in the $s u(2)$ sector, an all loop Hamiltonian that reproduces the proposed $s u(2)$ Bethe ansatz and the appropriate dispersion relations can be written down in terms of a strong coupling expansion of the Hubbard model 9 . Thus, as far as the large $N$ dynamics of single trace operators of the gauge theory is concerned, one has many indications that it is governed by an integrable (closed) spin chain.

Apart from single trace operators, the gauge theory also contains baryonic operators, whose engineering dimensions are of order $N$. These operators are constructed out of determinants and sub-determinants rather than traces [15, 16, 17. Such baryonic operators are of particular interest in the context of the AdS/CFT correspondence as they provide one with the gauge theory dual of non-perturbative D brane excitations i.e the so called giant gravitons of the dual string theory [18, 19, 21, 20, 22. The simplest example of a giant graviton is provided by a determinant operator constructed out of one of the three complex scalar fields $Z$

$$
\epsilon^{i_{1} \cdots i_{N}} \epsilon_{j_{1} \cdots j_{N}} Z_{i_{1}}^{j_{1}} \cdots Z_{i_{N}}^{j_{N}}
$$

This operator being built out of only one of the complex scalars is a BPS operator and does not undergo renormalization. The gravitational interpretation of this operator is that of a maximal D3-brane that saturates the angular momentum bound [19, 21, 20, 22, 23. Non-BPS excitations in the background of a giant graviton would correspond to open string excitations in the presence of the brane. The gauge theory provides us with a simple enough prescription for constructing the boundary theory dual of the open string degrees of freedom. The open string ending on the D3 brane can be built by replacing one of the $Z \mathrm{~s}$ in the determinant with a matrix product of local super-Yang-Mills fields [24, 4, 5, 25. A typical non-BPS su(2) operator would look like

$$
\epsilon^{i_{1} \cdots i_{N}} \epsilon_{j_{1} \cdots j_{N}} Z_{i_{1}}^{j_{1}} \cdots Z_{i_{N-1}}^{j_{N-1}}(W W Z Z Z W Z W Z \cdots Z Z W Z W)_{i_{N}}^{j_{N}} .
$$

Such operators, by virtue of not being protected by any non-renormalization theorems, would undergo operator mixing and hence have non-zero anomalous dimensions. The spectrum of anomalous dimensions of such operators gives one the gauge theory dual of the open string spectrum. Computing the spectrum of anomalous dimensions of such non-BPS maximal giants is the problem that we shall concern ourselves with in the present paper. As with various other issues concerning the AdS/CFT correspondence, it is important to analyze the problem in the large $N$ limit before addressing the problem in all its generality.

As it stands, it is not at all obvious that the computation of anomalous dimensions of giant graviton like operators simplifies in any way in the large $N$ limit. Processes that naively 
appear to be sub-leading order in $\frac{1}{N}$ may in fact turn out to be of order one as various $\frac{1}{N}$ suppressions may be compensated for by the $O(N)$ size of the operators. The fact that such counter-intuitive turn of events can come about in the study of Yang-Mills theories is rather well known from the study of the $\mathcal{N}=4 \mathrm{SYM}$ in the plane wave limit. There too one studies long operators that have large bare conformal dimensions and R-charge, J, and one has the result that if the ratio $\frac{J}{N^{2}}$ is held fixed then the usual notions of planarity do not apply any more [7. Non-planar contributions turn out to be of $O(1)$ in that limit. The study of operator mixing can still be reduced to a quantum mechanical problem, though the underlying quantum mechanics that one is faced with is not that of a quantum spin chain but that of a non-local many-body problem. Thus one of the pleasant features of the gauge theory, namely integrability is no longer present in this novel-large $N$ limit $^{1}$.

Though we shall not aspire to construct any new large $N$ limit where some parameter (other than $N$, such as the R-charge) is allowed to approach a critical value, and work completely within the framework of 't Hooft's large $N$ limit, the baryonic operators that we shall work with are indeed of order $N$. Thus we shall keep the lessons learnt from the study of the plane wave limit as a valuable caveat. From the studies of the dynamics of non-BPS giant gravitons of the gauge theory performed so far, the status of integrability is ambiguous. It was first shown in 24] that, at least at the one loop level, the anomalous dimensions of non-BPS giants are not of order one i.e although these operators have large bare conformal dimensions, their anomalous dimensions are small. Subsequently, more detailed one-loop analysis of non-BPS operators involving all six (real) SYM scalars was carried out in [4. In that paper, one loop mixing of operators such as

$$
\epsilon^{i_{1} \cdots i_{N}} \epsilon_{j_{1} \cdots j_{N}} Z_{i_{1}}^{j_{1}} \cdots Z_{i_{N-1}}^{j_{N-1}}\left(\phi^{i_{1}} \cdots \phi^{i_{l}}\right)_{i_{N}}^{j_{N}}
$$

where $\phi^{i}$ s can be any of the six scalars, was carried out. It was shown that the computation of one-loop anomalous dimensions of such operators can be reduced to the problem of diagonalizing the Minahan-Zarembo so(6) quantum spin chain, but with open boundary conditions. The open boundary conditions emerge naturally in the problem as the operators of interest are not traces, and the interaction of the scalar fields that form the chain(the matrix product of the $\phi$ fields in the above operator) with the $Z$ fields forming the brane lead to the open boundary conditions. The outcome of the analysis performed in [4] was the discovery that the open boundary conditions do not spoil the integrability of the Minahan-Zarembo spin chain. It was shown in that paper that the bulk and boundary $S$ matrices for the relevant $s o(6)$ spin chain satisfy the Yang-Baxter and reflection algebras: thus a necessary condition for

\footnotetext{
${ }^{1}$ In various other scenarios as well, where one takes into account non-planar splitting and joining of strings the notion of integrability, if any, appears to be far from obvious. We shall refer to [13, 14] for some recent developments in this direction.
} 
integrability is indeed satisfied at the one-loop level. However, already at the one loop level, the situation is different if one considers non-BPS non-maximal giants, which correspond to operators such as

$$
\epsilon_{i_{1} \cdots i_{k} j_{k+1} \cdots j_{N}}^{i_{1} \cdots i_{k} i_{k+1} \cdots i_{N}} Z_{i_{k+1}}^{j_{k+1}} \cdots Z_{i_{N-1}}^{j_{N-1}}(w)_{i_{N}}^{j_{N}}
$$

with $k>1$. $w$ stands for the matrix product formed out of two complex SYM scalars i.e. it is the $s u(2)$ spin chain. It was shown in [5] that the mixing of non-maximal giant gravitons of the above type formed out of two complex scalars can be formulated in the language of quantum spin chains, at least at the one loop level. The spin chain is nothing but the celebrated Heisenberg spin half model, but the boundary conditions generated by non-maximal giants are of a novel sort. The boundary interactions result in the spin chain being a dynamical one, i.e, the boundary interactions lead to fluctuations in the length of the chain. Though, it was possible to find the exact ground state of the spin chain in $[5]$ whether or not the chain is integrable is still an open issue ${ }^{2}$. Thus, integrability in $N=4$ SYM is not at all obvious once one ventures outside the realm of single trace operators.

Given this status quo, it is probably a reasonable goal to try and examine whether the spin chain interpretation of mixing of non-BPS giant gravitons holds beyond the one loop level and if it does it is of course interesting to investigate whether or not they can be diagonalized by Bethe ansatz techniques that have been so successful in the study of higher loop integrability of single trace/closed spin chain operators. In the present paper we shall take a step in that direction. The organization of the paper and the principal results reported in various sections are as follows.

In the next section we shall establish that the dynamics of non-BPS excitations in the background of a maximal giant graviton can be studied within the paradigm of open spin chains at the two loop level. In other words the mixing of non-BPS maximal giants will be shown to be described by open spin chains at the second order in the 't Hooft coupling. The analysis will be performed in two steps. We shall first resolve various combinatorial issues to work out the proper normalizations for various determinant and sub-determinant like operators. The guiding principle used in this exercise is that the various operators are required to have a free field two point function of order one. Once the combinatorial issues are settled, we shall proceed to study the mixing of these non-BPS giant gravitons. To that end we shall use the results of Beisert et al 3 ] where the two loop dilatation operator for the $s u(2)$ sector of the gauge theory was computed at finite $N$. Isolating the $O(1)$ part of the result of the action of the finite $N$ dilatation operator on the properly normalized operators

\footnotetext{
${ }^{2}$ The dynamical nature of the spin chain does not by itself rule out integrability. Even in the sector of closed chains e.g in the closed $s u(2 \mid 3)$ sector dynamical chains do appear 11 and they appear to be integrable 12. However, it is not known if the dynamical spin chains of interest in the sector of giant gravitons of the gauge theory enjoy similar integrable features.
} 
gives us the details of mixing of non-BPS giants at large $N$. We shall be able to show that at the two loop level non-BPS giants of the type(2) are closed under action of the dilatation operator. We shall also be able to interpret the process of mixing of such operators within the language of open spin chains; the open spin chain being the matrix product of various $s u(2)$ SYM fields that are contracted with the last pair of indices of the epsilon tensors in(2). We shall also be able to show that no new boundary conditions are generated at the two loop level. Thus the boundary conditions are the same as the ones discovered by Berenstein and collaborators in [4, which amount to the fact that the first and the last fields in the open chain(2) cannot be $Z \mathrm{~s}$. These boundary conditions emerge out of the realization that if a $Z$ field is placed at either end of the open spin chain, then the operator factorizes into a determinant and a trace, which is lower order in $\frac{1}{N}$. This fact may most easily be seen by going to a basis in which $Z$ is diagonal. Thus the boundary conditions for the problem at hand are not exactly the so called free boundary conditions, which would have corresponded to the case of the first and last spins being allowed to take any one of the two allowed values, but they are very similar.

Having a spin chain interpretation of the mixing of non-BPS giants at two loops is a gratifying outcome. The one loop open spin chain was already shown to be integrable in [4, and our analysis suggests that the study two loop operator mixing can be regarded as the analysis of a perturbation to an integrable open spin chain. The obvious question that comes to mind is whether or not the two loop spin chain Hamiltonian can be diagonalized by an appropriate Bethe ansarz. That, unfortunately does not appear to be the case. Before indulging in further details, it is probably worth emphasizing that even in the absence of integrability in the sense of the absence of a Bethe ansatz and a factorized $S$ matrix, the fact that the two loop operator mixing can be described by open spin chains is a major simplification in the study of the open string/giant graviton sector of the gauge theory. That is so because, as far as the computation of the spectrum of anomalous dimensions of operators that correspond to spin chains of finite length is concerned, the spin chain interpretation always reduces the problem to the process of diagonalization of a finite dimensional matrix, which can be achieved numerically. However, the lack of a two loop Bethe ansatz is a major drawback if one intends to study long coherent open string states constructed from the gauge theory and compare with the world-sheet analysis as was done in [24].

Having established the open spin chain description of the mixing of non-BPS giants, we shall devote the rest of the paper to a careful analysis of the integrability or the lack thereof of the two loop open spin chain. Although integrability of a given spin chain, even in a perturbative sense, is in general quite hard to establish, we shall take the pragmatic approach of Staudacher $[8$ and declare the spin chain to be integrable if one can successfully write down a set of Bethe equations for the spin chain. In the approach pioneered in 8 , the basic idea was to study the two magnon scattering problem, and find the appropriate two 
body $S$ matrix. Assuming factorized scattering, the two solution of the two body problem was then used to find the exact (in a perturbative sense) eigenstates, i.e the Bethe eigenstates of the general many body problem. An explicit construction of the scattering eigenstates of the $s u(2)$ dilatation operator and the corresponding Bethe equations, accurate to the third order in the 't Hooft coupling was also worked out by Staudacher in the same paper [8]. As far as the closed chains of the $s u(2)$ sector is concerned, the main complexity lay in the construction of the two magnon scattering matrix and the Bethe equations describing the two magnon scattering. As long as there is no global obstruction to the construction of the two particle $S$ matrix, one could assume factorized scattering and lift the solution of the two body problem to the case of an arbitrary number of magnons. One could then carry out further checks to ascertain that the solutions of the general many body problem do in fact reproduce the correct spectrum. In the $s u(2)$ sector, for instance, the spectrum obtained from the Bethe ansatz 8$]$ does match the spectrum obtained from the numerical lattice simulations of the corresponding spin chain [10. In a sense, the notion of integrability employed in this approach is the factorized nature of the scattering matrices for three or more magnon scattering processes.

However, in the presence of boundaries, there is a second notion of three body scattering, which is present even in the analysis of the two magnon problem. The presence of the boundary can lead to three body processes even if one is dealing with the dynamics of two magnons. Integrability in the presence of boundaries hence implies that these three body interactions take place in a way that is compatible with the separation of the scattering matrix into a bulk and a boundary part. Thus, even from the point of view of Staudacher's PABA apprach, the criterion of integrability is more stringent in the presence of non-periodic boundary conditions. We shall be able to demonstrate, in an explicit fashion that such three body processes, though absent at the one loop level, do start contributing at $O\left(\lambda^{2}\right)$. The algorithm that we employ to do this is the following one. We compute the bulk and boundary $S$ matrices from the study of the asymptotics of the two magnon problem. In other words, the study of the magnons scattering off each other far away from the boundaries gives us the bulk two body $S$ matrix of the spin chain and the situation when one of the magnons scatters off the boundary (while being far enough away from the other magnon) gives us the boundary $S$ matrix. From the knowledge of these scattering matrices, we can write down a set of Bethe equations. We then proceed to show that these Bethe equations follow from the requirement for a general ansatz for the two particle wave function to be an eigenstate of the spin chain Hamiltonian. Finally we demonstrate explicitly that the two body wave function that leads to the Bethe equations is not an eigenstate of the Hamiltonian. We interpret this to be evidence in favor of a breakdown of higher loop integrability in the open string sector of the gauge theory. Though the Bethe equations do not appear to be solutions of the two body Schrodinger equations, we can nevertheless utilize them to construct some 
exact eigenfunctions of the Hamiltonian. This construction is discussed at the very end of the third section.

From the point of view of the spin chain, we can also understand the potential breakdown of integrability in another way. The two loop dilatation operator that describes the mixing of non-BPS giants is the same as the one for closed chains as long as one stays away from the boundaries, i.e the bulk dilatation operator can be embedded in the Inozemtsev spin chain[26]. To the best of our knowledge, the only boundary conditions, other than the periodic ones, that are compatible with the integrability of the Inozemtsev chain are provided by reflective boundaries (see for example[27, 28, 29]). The boundary conditions that emerge out of the gauge theory analysis are not of that type, and as mentioned earlier, they are similar in spirit to the free boundary conditions. The Inozemtsev chain is not known to be integrable in the presence of free boundary conditions, and hence it is perhaps not surprising from this point of view that the two loop dilatation operator in question in the present paper does not appear to admit a consistent Bethe ansatz.

\section{Various Operators:}

The generic class of operators of interest to us are the ones that describe non-BPS excitations in the background of a maximal giant graviton. As mentioned earlier in the introduction, maximal BPS giants are protected operators formed out of determinants of a single complex scalar $Z$, and they define the vacuum state for the sector of interest to us.

$$
\mid 0>=\epsilon_{i_{1} \cdots i_{N}}^{j_{1} \cdots j_{N}} Z_{j_{1}}^{i_{1}} \cdots Z_{j_{N}}^{i_{N}}
$$

It is understood that

$$
\epsilon_{i_{1} \cdots i_{N}}^{j_{1} \cdots j_{N}}=\epsilon_{i_{1} \cdots i_{N}} \epsilon^{j_{1} \cdots j_{N}}
$$

A typical open string excitation corresponds to a state such as

$$
\mid k ; Z^{m} ; w_{1}, \cdots w_{N-k-m}>=\epsilon_{i_{1} \cdots i_{k} j_{k+1} \cdots j_{N}}^{i_{1} \cdots i_{k} i_{k+1} \cdots i_{N}} Z_{i_{k+1}}^{j_{k+1}} \cdots Z_{i_{k+m}}^{j_{k+m}}\left(w_{1}\right)_{i_{k+m+1}}^{j_{k+m+1}} \cdots\left(w_{N-k-m}\right)_{i_{N}}^{j_{N}}
$$

The $w_{i}$ s are matrix products of local Yang-Mills fields i.e they are the open string degrees of freedom. In the above operator, the indices of the epsilon tensor ( $k$ of them) which are contracted with each other indicate the departure from the maximal $R$ caharge bound, while, replacing a certain number of the $Z$ fields with the open strings, $w_{i}$, signal the departure form the BPS bound. As has been argued and demonstrated in 25, operators of the above kind do satisfy orthonormality conditions and it is possible to build a large $N$ Hilbert space using these open string degrees of freedom once the states are properly normalized. Rather than review the detailed combinatorial work presented in [25], which deals with the generic class 
of operators to which the above example belongs we shall focus on the particular operators of interest to us. We shall be interested in operators of the type

$$
\mid Z^{N-1}, w>
$$

which gives us the gauge theory description of a single open string ending on a maximal giant graviton.

$$
w_{j}^{i}=\left(w_{1} w_{2} \cdots w_{L}\right)_{j}^{i}
$$

Each of the 'bits' of the open string $w_{i}$ can take on two values $Z$ and $W$.

\subsection{Normalization:}

To be able to develop a consistent large $N$ expansion one needs to normalize the operators in a way that lends itself to a well defined large $N$ limit. We shall chose to normalize the operators by the free-field two point functions. It may be seen by a straightforward computation that

$$
\left\langle\bar{Z}^{N-1}(x), \bar{w}(x) \mid Z^{N-1}(0), w(0)\right\rangle \sim[(N-1) !]^{3} N^{L+1} \frac{1}{|x|^{N+L-1}}
$$

Hence, it is reasonable to normalize the states as

$$
\frac{1}{\sqrt{[(N-1) !]^{3} N^{L+1}}} \mid Z^{N-1}, w>
$$

As mentioned in the introduction, with this normalization one is led immediately to dirichlet boundary conditions for the open spin chain $w$. It may be easily verified that if either $w_{1}$ or $w_{L}$ is equal to $Z$, then the state factorizes into a product of a determinant and a single trace [4, 25]. With our chosen normalization, such a state would turn out to be sub-leading order in the large $N$ limit. Thus, one has the Dirichlet boundary conditions that, neither the first or the last impurity fields can be of the $Z$ type.

\subsection{The Open Spin Chain:}

The two loop dilatation operator of $\mathcal{N}=4 \mathrm{SYM}$ in the $s u(2)$ sector is 3 ]

$$
D=D_{0}+\frac{\lambda}{N} D_{1}+\frac{\lambda^{2}}{N^{2}} D_{2}+O\left(\lambda^{3}\right)
$$

where

$$
D_{0}=\operatorname{tr}\left(A^{\dagger Z} A_{Z}+A^{\dagger W} A_{W}\right)
$$




$$
\begin{aligned}
& D_{1}=-\operatorname{tr}\left[A^{\dagger Z}, A^{\dagger W}\right]\left[A_{Z}, A_{W}\right] \\
D_{2}= & \operatorname{tr}\left(\frac{1}{2}\left[A_{Z}, A_{W}\right]\left[A^{\dagger Z},\left[A_{Z},\left[A^{\dagger Z}, A^{\dagger W}\right]\right]\right]\right. \\
+ & \frac{1}{2}\left[A_{Z}, A_{W}\right]\left[A^{\dagger W},\left[A_{W},\left[A_{Z}^{\dagger}, A_{W}^{\dagger}\right]\right]\right] \\
+ & {\left.\left[A^{\dagger Z}, A^{\dagger W}\right]\left[A_{Z}, A_{W}\right]\right), }
\end{aligned}
$$

and

$$
\lambda=\frac{g_{Y M}^{2} N}{8 \pi^{2}} .
$$

It is understood, that one has mapped the local composite $(s u(2))$ operators to states of the matrix model Hamiltonian. For example,

$$
\operatorname{Tr}(Z Z W W \cdots W) \Longleftrightarrow \operatorname{Tr}\left(A^{\dagger Z} A^{\dagger Z} A^{\dagger W} \cdots A^{\dagger W}\right) \mid 0>
$$

and that operator mixing is mapped to the action of the dilatation operator, thought of as a matrix model Hamiltonian, on various states like the one depicted above. When the dilatation operator acts a giant graviton like state such as $\mid Z^{N-1}, w>$ the following possibilities may occur.

(1:) All the annihilation operators of $D_{1}$ and $D_{2}$ may act on $w$.

(2:) Some of the annihilation operators may act on $w$ and others on the $Z$ fields that form the brane.

In the first instance, one has the standard result that the action of the annihilation operators on consecutive fields present in $w$ can be interpreted as the action of a quantum spin chain Hamiltonian on a spin chain state 3, 30. The action of the annihilation operators on non-consecutive fields can be shown to be sub-leading order in $\frac{1}{N}$. Such actions always spit up the spin chain into multiple chains, thus transforming the state with a single string excitation in the D3 background to one involving multiple open string excitations. Such states are mutually orthogonal (see 25] for the combinatorial details). Hence they can be ignored for the purposes of large $N$ computations. The spin chain that results from the 'bulk' action of the dilatation operators on consecutive fields present in $w$ is the same as the one that one has in the study of single trace operators [3], only the boundary conditions are different. As mentioned previously, the boundary conditions consist of the condition that neither the first or the last fields of the spin chain can be a $Z$.

When the dilatation operator acts on two $Z$ fields from the brane, the result can be easily shown to be sub-leading order in $\frac{1}{N}$. However, special care is required to analyze the result of the action of the dilatation operator on two consecutive fields in $w$ and a single $Z$ field 
from the brane. A careful analysis shows that there are no $O(1)$ contributions from such actions of the dilatation operator. Indeed, all the terms so generated are given below along with the leading powers of $\frac{1}{N}$ by which they are suppressed. In the following list, $w_{1}$ stands for a single field, $w_{2}$ represents a generic 'word' formed out of two fields and $w_{c}$ is a word of length $|w|-2:|w|$ being the length of $w$. The second set of states multiplying the fifth and lower terms in the table are single trace states which result upon the action of the dilatation operator on the giant graviton state.

$$
\left|\begin{array}{c|c}
\mid Z^{N-2}, w_{1}, w_{c} w_{2}> & \frac{1}{\sqrt{N}} \\
\mid Z^{N-2}, w_{3}, w_{c}> & \frac{1}{\sqrt{N}} \\
\mid Z^{N-2}, w_{2}, w_{1} w_{c}> & \frac{1}{\sqrt{N}} \\
\mid 1, Z^{N-2}, w_{3} w_{c}> & \frac{1}{\sqrt{N}} \\
\left|1, Z^{N-2}, w_{c}>\right| w_{3}> & \frac{1}{\sqrt{N}} \\
\left|1, Z^{N-2}, w_{2} w_{c}>\right| w_{1}> & \frac{1}{\sqrt{N}} \\
\left|1, Z^{N-2}, w_{1} w_{c}>\right| w_{2}> & \frac{1}{\sqrt{N}} \\
\left|Z^{N-2}, w_{2}, w_{c}>\right| w_{1}> & \frac{1}{N} \\
\left|1, Z^{N-2}, w_{2} w_{c}>\right| w_{1}> & \frac{1}{\sqrt{N}} \\
\left|Z^{N-2}, w_{1}, w_{c}>\right| w_{2}> & \frac{1}{\sqrt{N}}
\end{array}\right|
$$

We can now write down the formula for the two loop large $N$ open spin chain. As mentioned in the preceding discussion the two loop Hamiltonian for the open string sector is the same as the one for single trace operators, the only thing that is is different is the boundary condition. To incorporate the boundary conditions in the formula for the Hamiltonian we shall use the operators $Q_{i}(Z)$, which were introduced in [4. These operators are defined by their actions on the spin chain states as foillows:

$$
\begin{aligned}
Q_{i}^{Z} \mid \cdots Z \cdots> & =0 \\
Q_{i}^{Z} \mid \cdots W \cdots> & =\mid \cdots W \cdots>.
\end{aligned}
$$

In the above equations, $Z, W$ are located at the 'i'th lattice site. i.e. the operator $Q_{i}(Z)$ checks if the 'i'th spin is a $Z$. If it is so then it annihilates the entire state. Using these operators, the open spin chian Hamiltonian can be written as

$$
\begin{aligned}
H= & \sum_{l=1}^{J-1}(1-4 g) Q_{1}^{Z} Q_{J}^{Z}\left(I-P_{l, l+1}\right) Q_{1}^{Z} Q_{J}^{Z} \\
& +g \sum_{l-1}^{J-2} Q_{1}^{Z} Q_{J}^{Z}\left(I-P_{l, l+2}\right) Q_{1}^{Z} Q_{J}^{Z}
\end{aligned}
$$


To get to the above form, we have divided out the formula for the Hamiltonian by $\lambda$ and used

$$
g=\frac{\lambda}{2} .
$$

It is of course assumed that the length of the chain is $J$ and the Hamiltonian acts on all the spin sites. Since the first and the last sites cannot have $Z$ fields, one could write down the Hamiltonian is a slightly different form as well. We could take a state of the generic form

$$
|W \cdots W\rangle,
$$

where one could have impurity $(Z)$ fields inserted between two $W$ fields. In the previous formulation, the spin chain included the boundary fields, but we could just as well modify the definition of the spin chain to include only the fields contained inside the two boundary $W$ fields, and write the effect of the interaction of the boundary fields with the spin chain separately. In other words, we shall define the second field of $w$ to be first site of the spin chain and the penultimate field of $w$ to be the last site. Thought of in this way, the spin chain Hamiltonian reads as

$$
\begin{array}{r}
H=\sum_{l=1}^{L-1}(1-4 g)\left(I-P_{l, l+1}\right)+g \sum_{l=1}^{L-2}\left(I-P_{l, l+2}\right) \\
\quad(1-4 g) q_{1}^{Z}+g q_{2}^{Z}+(1-4 g) q_{L}^{Z}+g q_{L-1}^{Z}
\end{array}
$$

It is to be understood that

$$
q_{i}^{Z}=\left(1-Q_{i}^{Z}\right),
$$

i.e $q_{i}^{Z}$ checks if the field at the $i$ th site is $Z$, and if it is so, it acts as the identity operator. In case this condition is not satisfied, $q_{i}^{Z}$ annihilates the spin chain. $L$ is now to be regarded as the effective length of the spin chain i.e the number for fields between the boundary $W$ fields i.e.

$$
L=|w|-2 .
$$

\subsection{Bethe Ansatz:}

Let us begin by deriving the one loop Bethe ansatz for the open spin chain. Short ranged spin chains of the Heisenberg type with various boundary conditions have been solved using both the coordinate and algebraic Bethe ansatz techniques in the past 31, 32, 33. In the present paper, we shall adhere to the coordinate Bethe ansatz approach as it generalizes easily to higher loops. An excellent discussion of coordinate Bethe ansatz solutions to short 
ranged spin chains can be found in 31, 32], and we shall adhere to the approach presented in these papers in this present analysis.

The one magnon state $\mid \Psi_{1}>$ can be taken to be of the form:

$$
\left|\Psi_{1}>=\sum_{x=1}^{L} \Psi(x)\right| x>.
$$

If one takes the state to be a superposition of incoming and outgoing plane waves, with

$$
\Psi(x)=A(k) e^{i k x}-A(-k) e^{-i k x},
$$

then it is quite easy to see that the state is an eigenstate of the Hamiltonian with energy

$$
E(k)=4 \sin ^{2}\left(\frac{k}{2}\right) .
$$

It is also straightforward to verify that for the plane wave state to be an eigenstate, the following boundary conditions, which are implied by scattering at the boundary, must hold.

$$
\begin{aligned}
\Psi(0) & =0 \\
\Psi(L+1) & =0 .
\end{aligned}
$$

These conditions can also be written down as

$$
\begin{aligned}
& \alpha(-k) A(k)-\alpha(k) A(-k)=0 \\
& \beta(k) A(k)-\beta(-k) A(-k)=0 .
\end{aligned}
$$

The above conditions lead to the following compatibility condition

$$
\alpha(k) \beta(k)=\alpha(-k) \beta(-k)
$$

with $\alpha, \beta$ given by

$$
\begin{aligned}
\alpha(-k) & =1 \\
\beta(k) & =e^{i(L+1) k} .
\end{aligned}
$$

The solution to the compatibility condition sets the allowed range of values for the momenta to be

$$
k=\frac{n \pi}{L+1} .
$$


This is the complete solution to the one magnon scattering problem, which gives us the one-magnon spectrum(2631) and the boundary scattering matrix $\frac{A(k)}{A(-k)}$.

Having determined the boundary $S$ matrix by solving the one-magnon problem, one can now proceed to study the two magnon scattering problem. The two magnon state can be taken to be of the form

$$
\left|\Psi_{2}>=\sum_{x<y} \Psi(x, y)\right| x, y>
$$

with

$$
\Psi(x, y)=\sum_{p} \sigma(p) A\left(k_{i}, k_{2}\right) e^{i\left(k_{1} x_{1}+k_{2} x_{2}\right)} .
$$

The above sum is over all permutations and negations $(\mathrm{p})$ of the momenta. $\sigma(p)= \pm 1$ and it changes sign when the signs of when one of the signs of the moments are changed or when the momenta are permuted in pairs. This two magnon state is an eigenstate of the spin chain Hamiltonian provided the following conditions are satisfied.

$$
\begin{aligned}
A\left(k_{1}, k_{2}\right) s\left(k_{1}, k_{2}\right)-A\left(k_{2}, k_{1}\right) s\left(k_{2}, k_{1}\right) & =0 \\
A\left(k_{1}, k_{2}\right) \alpha\left(-k_{1}\right)-A\left(-k_{1}, k_{2}\right) \alpha\left(k_{1}\right) & =0 \\
A\left(k_{1}, k_{2}\right) \beta\left(k_{2}\right)-A\left(k_{1},-k_{2}\right) \beta\left(-k_{2}\right) & =0
\end{aligned}
$$

In the first equation

$$
s\left(k_{1}, k_{2}\right)=1-2 e^{i k_{2}}+e^{i\left(k_{1}+k_{2}\right)} .
$$

The three equations above require some explanation. The first of the three equation comes from the requirement that two body scattering, that takes place when the impurities appear next to each other, happens in a way such that the state remains an eigenstate of the Hamiltonian. The other two equations follow from scattering at the boundary, i.e by considering the scenario that one of the particles is at the boundary and by requiring the coefficient of this state to be such that it still remain an eigenstate of the Hamiltonian. In a sense we can see, directly from these equations that the boundary conditions are compatible with integrability at the one loop level. That follows from the observation that the boundary scattering equations involve only one of the two momenta. In other words, the boundary does not introduce any new 'three body' interactions. For example, if one considered the special case when the two particles sit next to each other at the boundary, there could in principle have been potential three body interactions; the third body being the boundary. However, the above conditions tell us that this is not the case at the one loop level, and the two boundary equations are all that one needs for all positions of the second impurity. We shall soon see that this is not the case at the two loop level. 
The situation is more transparent in position space. For any pair of well separated lattice sites away from the boundary,

$$
H|x, y(>x+1)>=4| x, y>-|x-1, y>-| x+1, y>-|x, y-1>-| x, y+1>
$$

With the choice of the pane wave ansatz, and this action of the Hamiltonian, it is clear that the coefficient of $\mid x, y(>x+1)>$, once the Hamiltonian has acted on it, is $\left(4 \sin ^{2}\left(\frac{k_{1}^{2}}{2}\right)+\right.$ $\left.4 \sin ^{2}\left(\frac{k_{2}^{2}}{2}\right)\right) \Psi(x, y)$. However, for the coefficient of $\mid x, y(=x+1)>$ to come out right, one has to impose the condition

$$
2 \Psi(x, x+1)-\Psi(x+1, x+1)-\Psi(x, x)=0
$$

which leads to the scattering condition; the first equation in (34). Once this equation is satisfied, the effects of the boundary impose the further constraint

$$
\begin{array}{r}
\Psi(0, y)=0 \\
\Psi(x, L+1)=0
\end{array}
$$

which translate into the second and third equations in (34) respectively. What is notable is that once the two particle scattering equations are satisfied, for some choice of $\Psi(x, y)$, the boundary conditions on $\Psi(x, y)$ are the same as the one magnon case. For instance, the left boundary condition (the first of the two equations above) is the same as the condition satisfied by $\Psi(x)$ in the one-magnon problem for all values of $y$. Most importantly, the special situation when $x=1, y=2$ does not introduce any new conditions on the wave function. Thus the bulk and boundary scattering problems separate out. This is nothing but an explicit (and perhaps pedagogical) way of realizing that the boundary conditions are compatible with integrability of the spin chain.

Having the three equations at out disposal, we can now derive the Bethe equations that determine the momenta. As in the one magnon case, the Bethe equations are derived as the compatibility conditions implied by the three equations. Various ways of expressing $A\left(k_{1}, k_{2}\right)$ in terms of $A\left(k_{2}, k_{1}\right)$ lead to the requirement 32 .

$$
\frac{\alpha\left(k_{1}\right) \beta\left(k_{1}\right)}{\alpha\left(-k_{1}\right) \beta\left(-k_{1}\right)}=\frac{S\left(-k_{1}, k_{2}\right)}{S\left(k_{1}, k_{2}\right)}
$$

where

$$
S\left(k_{1}, k_{2}\right)=s\left(k_{1}, k_{2}\right) s\left(k_{2},-k_{1}\right)
$$

There is a similar equation where $k_{1}$ is replaced by $k_{2}$ and vice-versa. These results can be generalized to the case of an arbitrary number of magnons. In the case of $m$ magnons the 
Bethe equations read as

$$
\frac{\alpha\left(k_{i}\right) \beta\left(k_{i}\right)}{\alpha\left(-k_{i}\right) \beta\left(-k_{i}\right)}=\prod_{j(\neq i)=1}^{m} \frac{S\left(-k_{i}, k_{j}\right)}{S\left(k_{i}, k_{j}\right)} .
$$

\section{The Two Loop Analysis:}

Bethe ansatz for the $s u(2)$ open spin chain decribed in the previous section is essentially implied in the analysis carried out in 4 . where the one loop bulk and boundary $S$ matrices of the full so(6) spin chain were computed. The above analysis reconfirms and reproduces the $s u(2)$ sector of the results reported in 4 from a coordinate Bethe ansatz point of view and also sets up the techniques and conventions that will be applied to the two loop analysis, to which we shall devote the rest of the paper. The two loop large $N$ dilatation operator is

$$
\begin{array}{r}
H=\sum_{l=1}^{L-1}(1-4 g)\left(I-P_{l, l+1}\right)+g \sum_{l=1}^{L-2}\left(I-P_{l, l+2}\right) \\
\quad(1-4 g) q_{1}^{Z}+g q_{2}^{Z}+(1-4 g) q_{L}^{Z}+g q_{L-1}^{Z} .
\end{array}
$$

As was the case in the analysis of single trace operators, we do not expect the two loop spin chain to be exactly integrable. However, in the analysis of closed spin chains, it was possible to establish a perturbative integrablity of the large $N$ dilatation operator. In other words, it was possible to construct a Bethe ansatz for the eigenstates of the two loop Hamiltonian which was accurate to $O(g)$ [ . It is thus reasonable to examine if the two loop large $N$ dilatation operator exhibits this perturbative notion of integrability in the background of giant gravitons.

The method of the perturbative assymptotic Bethe ansatz (PABA) advocated by Staudacher in [8] provides one with a very direct and transparent method for checking whether or not a given spin chain, whose Hamiltonian can be expressed as a power series in some coupling constant (in our case $g$ ), admits of a Bethe ansatz order by order in perturbation theory. This method was successfully applied to the study of the mixing of single trace $s u(2)$ operators of the gauge theory up to three loops in perturbation theory. In the sector of single trace operators, the large $N$ dilatation operator maps to closed spin chains with periodic boundary conditions. For such spin chains, there are no boundaries, and the magnons only scatter off each other. Establishing the existence of a PABA for the closed spin chains thus consists of a two step process.

(1:) Finding the two body $S$ matrix for two magnon scattering while allowing for the $S$ matrix to have an appropriate $g$ dependence such that the two magnon Bethe state is an eigenstate of the Hamiltonian in the perturbative sense mentioned above. 
( 2:) Generalizing the two body solution to a solution of the many body problem using the kind of reasoning used at the end of the previous section, which basically amounts to assuming factorized scattering for the problem.

For the problem at hand, the only difference between the two loop Hamiltonian(42) and the corresponding spin chains describing the two loop dilatation operator for single trace states lies in the boundary conditions and the boundary terms(the second line in (42)). Thus, when the magnons are far away from the boundaries, the scattering between the magnons is described by the same $S$ matrix that appears in the case of the closed spin chains, which was already found in $[\underline{8}$. Thus, for the purposes of the present analysis, we can borrow from 8 the formula for the two loop bulk two magnon scattering matrix. However, for the present problem, we also need to perform a PABA to account for the scattering of the magnons from the boundaries i.e compute the boundary scattering matrix accurate to two loop order. As in the case of the one-loop analysis performed earlier in the paper, the boundary scattering matrix, will be found by solving the one magnon problem. To clinch the issue of perturbative integrabilty in analysis of the open spin chain would require a demonstration that the Bethe state formed by merging the two asymptotic solutions is an eigenstate of the Hamiltonian. To put it differently, we shall have to check that two magnon state that has as the bulk $S$ matrix the one found in 8 , and as the boundary $S$ matrix the appropriate solution of the one magnon problem is an eigenstate of the Hamiltonian. This unfortunately is not the case. We shall now proceed to substantiate these comments by explicit computations in the following sections.

\subsection{One Magnon Problem:}

As in the one-loop case, we shall first compute the boundary $S$ matrix of the spin chain by solving the one-magnon problem. An important distinction from the one-loop analysis is that the Bethe wave function is not simply a superposition of plane waves. We shall also have to allow for some exponential decay of the wave function around the boundaries. The analysis below can be thought of as the two loop PABA for the one magnon problem. Although this is ostensibly a one-body problem, the presence of the boundary induces a two body effect through the interactions of the impurity with the boundary. Just like the two body problem in Staudacher's PABA [ 8 required the introduction of an exponential decay of the two body wave function for the Bethe ansatz to be consistent, the one magnon problem in the present analysis requires that the one body wave function be allowed to have an exponential decay around the boundaries. The Bethe ansatz that incorporates this requisite feature can be 
summarized in the following form for the one magnon wave function:

$$
\left|\Psi>=\sum_{x=1}^{L} \Psi(x)\right| x>
$$

where

$$
\Psi(x)=A(k) e^{i k x}-A(-k) e^{-i k x}+a(k) g^{x}+b(k) g^{L-x}
$$

As is evident from the above formula, the third and the fourth terms account for the exponential decay of the wave function; the strength of the decay being given by the 't Hooft coupling constant. To avoid having to write factors involving the momenta, let us call

$$
\begin{gathered}
A(k) e^{i k x}-A(-k) e^{-i k x}=\psi(x) \\
a(k)=\phi(1) \\
b(k)=\phi(L)
\end{gathered}
$$

As in the one loop analysis, when the impurity is sufficiently far away from the boundary i.e for any $2<x<L-2$ we have the condition:

$$
(1-4 g)(2 \psi(x)-\psi(x-1)-\psi(x+1))+g(2 \psi(x)-\psi(x-2)-\psi(x+2))=E \Psi(x)
$$

with

$$
E(k)=4 \sin ^{2}\left(\frac{k}{2}\right)-16 g \sin ^{4}\left(\frac{k}{2}\right)=E_{1}(k)+g E_{2}(k) .
$$

Special care is needed to analyze the situations $x=2,1, L, L-1$. The coefficient of $\mid x=2>$, after the action of the Hamiltonian on the Bethe wave function is

$$
(2-4 g)(2 \psi(2)-\psi(1)-\psi(3))+g(2 \psi(2)-\psi(4))-g \phi(1) .
$$

Thus we have the condition

$$
\psi(0)=\phi(1)
$$

Similarly, the coefficient of $\mid x=1>$ is

$$
(1-4 g)(2 \psi(1)-\psi(2))+g(\psi(1)-\psi(3))+2 g \phi(1)
$$

This leads to the condition:

$$
(1-4 g) \psi(0)+g \psi(-1)-g \psi(1)+2 g \phi(1)=g E_{1}(k) \phi(1)
$$


where $E_{1}(k)=4 \sin ^{2}\left(\frac{k}{2}\right)$. There are similar equations that follow from the scattering at the right boundary. These equations may be written in a way that is at par with the one loop equations(28) i.e

$$
\begin{aligned}
& A(k) \alpha(-k)-A(-k) \alpha(k)=0 \\
& A(k) \beta(k)-A(-k) \beta(-k)=0 .
\end{aligned}
$$

$\alpha(\beta)$ determine the two loop left (right) boundary scattering amplitudes and are given by

$$
\begin{aligned}
\alpha(-k) & =(1-2 g)+g e^{-i k}-g e^{i k}-g E_{1}(k) \\
\beta(k) & =e^{i k(L+1)}\left((1-2 g)+g e^{i k}-g e^{-i k}-g E_{1}(k)\right) .
\end{aligned}
$$

As at the one loop level, the compatibility conditions $\alpha(k) \beta(k)=\alpha(-k) \beta(-k)$ determine the momenta to be

$$
k=\frac{n \pi-\theta(k)}{L+1}
$$

where

$$
\theta(k)=\tan ^{-1}\left[\frac{2 g \sin (k)}{(1-2 g)-g E_{1}(k)}\right] .
$$

It is understood that the expression for $\theta$ is to be regarded as an approximate expression accurate to $O(g) . \theta(k)$ gives us the two loop correction from the boundary scattering to the allowed values of momenta. This completes the Bethe ansatz for the one magnon probelm.

\subsection{Two Magnon Problem:}

Having solved the one magnon problem, we can now analyze the case of two magnons. Once again, we shall have to propose a Bethe ansatz, that accounts for the exponential decay of the wave functions when the magnons interact with each other or with the boundary. The appropriate PABA can be summarized in the following two body wave function

$$
\left|\Psi>=\sum_{x<y} \Psi(x, y)\right| x, y>=\sum_{x<y} \psi(x, y)\left|x, y>+g \sum_{y>1} \phi(1, y)\right| 1, y>+g \sum_{x<L} \tilde{\phi}(x, L) \mid x, L>.
$$

As in Saudacher's PABA [8] we shall let $\psi\left(k_{1}, k_{2}\right)$ be a superposition of plane waves, with some exponential decay that takes place when the magnons interact with each other, while $\phi, \tilde{\phi}$ will measure the amplitude of decays around the boundaries. More specifically, we shall have

$$
\psi\left(x_{1}, x_{2}\right)=\sum_{p} \sigma(p) e^{i\left(k_{1} x_{1}+k_{2} x_{2}\right)}\left(A\left(k_{1}, k_{2}\right)+B\left(k_{1}, k_{2}\right) g^{\left|x_{1}-x_{2}\right|}\right)
$$


Once again, for two magnons at $x_{1}>2$ and $L-2>x_{2}>x_{1}+2$, the coefficient of $\mid x_{1}, x_{2}>$ after the action of the Hamiltonian is

$$
\begin{array}{r}
(1-4 g)\left(4 \psi\left(x_{1}, x_{2}\right)-\psi\left(x_{1}-1, x_{2}\right)-\psi\left(x_{1}+1, x_{2}\right)-\psi\left(x_{1}, x_{2}-1\right)-\psi\left(x_{1}, x_{2}+1\right)\right) \\
+g\left(4 \psi\left(x_{1}, x_{2}\right)-\psi\left(x_{1}-2, x_{2}\right)-\psi\left(x_{1}+2, x_{2}\right)-\psi\left(x_{1}, x_{2}-2\right)-\psi\left(x_{1}, x_{2}+2\right)\right) \\
=E\left(k_{1}, k_{2}\right) \psi\left(x_{1}, x_{2}\right)=\sum_{i=1,2}\left(4 \sin ^{2}\left(\frac{k_{i}}{2}\right)-16 g \sin ^{4}\left(\frac{k_{i}}{2}\right)\right) \psi\left(x_{1}, x_{2}\right)
\end{array}
$$

At the special situation when $x_{2}=x_{1}+2$, one has the condition

$$
2 \psi(x, x+2)-\psi(x+2, x+2)-\psi(x, x)=0,
$$

while when $x_{2}=x_{1}+1$, one has the condition

$$
\begin{array}{r}
g\left(\psi\left(x_{1}+2, x_{2}\right)+\psi\left(x_{1}, x_{2}-2\right)-\psi\left(x_{1}-1, x_{2}-1\right)-\psi\left(x_{1}+1, x_{2}+1\right)\right) \\
(4 g-1)\left(2 \psi\left(x_{1}, x_{2}\right)-\psi\left(x_{1}+1, x_{2}\right)-\psi\left(x_{1}, x_{2}-1\right)\right)=0
\end{array}
$$

This part of the computation is the same as the PABA for periodic spin chains carried out by Staudacher in 8 . The above conditions serve to fix the function $B\left(k_{1}, k_{2}\right)$ and to determine the bulk scattering matrix. However the boundary processes have to be treated separately which we shall now proceed to do. Now, for any $x_{2}$, the condition obtained from looking at the coefficient of $\mid 2, x_{2}>$ is

$$
\psi\left(0, x_{2}\right)=\phi\left(1, x_{2}\right) .
$$

One also has a similar condition at the right boundary; namely

$$
\psi\left(x_{1}, L+1\right)=\phi\left(x_{1}, L\right)
$$

Furthermore, for any $x_{2}>2$ and $x_{1}<L-1$ the coefficients of $\mid 1, x_{2}>$ and $\mid x_{1}, L>$ lead to following two boundary scattering equations respectively.

$$
\begin{gathered}
A\left(k_{1}, k_{2}\right) \alpha\left(-k_{1}\right)-A\left(-k_{1}, k_{2}\right) \alpha\left(k_{1}\right)=0 \\
A\left(k_{1}, k_{2}\right) \beta\left(k_{2}\right)-A\left(k_{1},-k_{2}\right) \beta\left(-k_{2}\right),
\end{gathered}
$$

with $\alpha, \beta$ given by(53). Equations(59, 60, 61, 62, 63) completely fix all the undertermined functions in the problem. Indeed, they also lead to the Bethe equations for the momenta

$$
\frac{\alpha\left(k_{i}\right) \beta\left(k_{i}\right)}{\alpha\left(-k_{i}\right) \beta\left(-k_{i}\right)}=\prod_{j \neq i} S\left(-k_{i}, k_{j}\right) S\left(k_{j}, k_{i}\right) .
$$


$\alpha$ and $\beta$ are given by (53), while the bulk $S$ matrix is the solution of (59606162). It is the same as the one presented in $8,6,9]$.

$$
S\left(p, p^{\prime}\right)=\frac{\phi(p)-\phi\left(p^{\prime}\right)+i}{\phi(p)-\phi\left(p^{\prime}\right)-i}
$$

with the phase shift (thought of as an expression accurate to order $\lambda$ for the present problem) being given by

$$
\phi(p)=\frac{1}{2} \cot \left(\frac{p}{2}\right) \sqrt{1+8 \lambda \sin ^{2}\left(\frac{p^{2}}{2}\right)} .
$$

This situation is very much at par with what one had at one loop(34). The boundary interactions so far have not introduced any three-body interactions. The effect of the left (right) boundary scattering on $\psi\left(x_{1}, x_{2}\right)$ produces the same algebraic equations satisfied by $\psi(x)$ in the one magnon problem. Thus in the analysis so far, the boundary has not 'seen' the second particle. That is not surprising as we have not analyzed the only situation that can lead to a three body process, which is when both the impurities are near the boundary: i.e we need to study the coefficient of the state $\mid 1,2>$ after the action of the Hamiltonian on the Bethe wavefunction. This is the only scenario (at this loop order) in which all the three 'particles', the two impurities and the boundary can interact with each other. Thus the spin chain will be perturbatively integrable, if the coefficient of $\mid 1,2>$ does not lead to any new conditions. Unfortunately, that is not the case. It is a straightforward exercise to see that the coefficient of $\mid 1,2>$ leads to the condition

$$
\sum_{p} \sigma(p) A\left(k_{1}, k_{2}\right) \tilde{\alpha}\left(-k_{1}, k_{2}\right)=0
$$

where

$$
\tilde{\alpha}\left(-k_{1}, k_{2}\right)=e^{i k_{2}}\left((1-4 g)+g\left(-e^{i k_{1}}+2 e^{-i k_{2}}+e^{-i k_{1}}-E_{1}\left(k_{1}\right)\right)\right)
$$

Similarly, at the right boundary, the coefficient of $\mid L-1, L>$ leads to

$$
\sum_{p} \sigma(p) A\left(k_{1}, k_{2}\right) \tilde{\beta}\left(k_{1}, k_{2}\right)=0
$$

where

$$
\tilde{\beta}\left(k_{1}, k_{2}\right)=e^{i\left(k_{1}(L-1)+k_{2}(L+1)\right)}\left((1-4 g)+g\left(e^{i k_{2}}-e^{-i k_{2}}+2 e^{i k_{1}}-E_{1}\left(k_{2}\right)\right)\right)
$$

These are new conditions which imply that the Bethe state is not an eigenstate of the Hamiltonian even after one finds bulk and boundary S matrices which are solutions of the 
two body Schrodinger equations. The presence of both the momenta $k_{1}, k_{2}$ in boundary scattering phase shifts $\tilde{\alpha}, \tilde{\beta}$ in the above equations is the signature of three body interactions that prevent the separation of the scattering matrix into a bulk and a boundary part. The extra three body interaction coming from the special possibility of both the particles being close to the boundary thus shows us that the conventional Bethe ansatz does not quite work in the case of the present problem.

Though the problem does not appear to be integrable, at least from the PABA point of view, we can nevertheless use the Bethe equations to construct some exact two particle eigenstates. This can be carried out by allowing for a linear superposition of the the Bethe wave functions of the kind discussed above with various values of the momenta. Instead of(56), we can look for a wave function of the type

$$
\Psi(x, y)=\Psi(x, y)+\Gamma \Psi^{\prime}(x, y)+\Delta \Psi^{\prime \prime}(x, y),
$$

where $\Psi^{\prime}, \Psi^{\prime \prime}$ have exactly the same functional form as $\Psi$, but they are chosen to have different momenta, $k_{1}^{\prime}, k_{2}^{\prime}$, and $k_{1}^{\prime \prime}, k_{2}^{\prime \prime}$ respectively. The functions are also chosen to satisfy the scattering equations(5960616263). Furthermore, we shall choose the three pairs of momenta to be related by

$$
\sum_{i=1,2} E\left(k_{i}\right)=\sum_{i=1,2} E\left(k_{i}^{\prime}\right)=\sum_{i=1,2} E\left(k_{i}^{\prime \prime}\right)
$$

with $E(k)$ given by (47). We can now fix the two undertermined functions of the momenta $\Delta, \Gamma$ by requiring the state to be an eigenstate of the Hamiltonian. Clearly, the equations that need to be satisfied by $\Gamma$ and $\Delta$ follow from (6769). They are

$$
\sum^{\prime} A\left(k_{1}, k_{2}\right) \tilde{\alpha}\left(-k_{1}, k_{2}\right)+\Gamma \sum^{\prime}\left(A\left(k_{1}^{\prime}, k_{2}^{\prime}\right) \tilde{\alpha}\left(-k_{1}^{\prime}, k_{2}^{\prime}\right)\right)+\Delta \sum^{\prime}\left(A\left(k_{1}^{\prime \prime}, k_{2}^{\prime \prime}\right) \tilde{\alpha}\left(-k_{1}^{\prime \prime}, k_{2}^{\prime \prime}\right)\right)=0
$$

and

$$
\sum^{\prime} A\left(k_{1}, k_{2}\right) \tilde{\beta}\left(k_{1}, k_{2}\right)+\Gamma \sum^{\prime}\left(A\left(k_{1}^{\prime}, k_{2}^{\prime}\right) \tilde{\beta}\left(k_{1}^{\prime}, k_{2}^{\prime}\right)\right)+\Delta \sum^{\prime}\left(A\left(k_{1}^{\prime \prime}, k_{2}^{\prime \prime}\right) \tilde{\beta}\left(k_{1}^{\prime \prime}, k_{2}^{\prime \prime}\right)\right)=0
$$

In the above equations the 'primed' sums refer to summations over all permutations and negations of momenta as we have carried out in the rest of the paper; i.e $\sum^{\prime} \equiv \sum_{p} \sigma(p)$. These equations can indeed be solved, allowing us to construct some special eigenstates of the Hamiltonian. In other words, the two body problem is solvable in a sub-space of the Hilbert space spanned by the Bethe wavefunctions. In the presence of integrability, every Bethe state corresponding to any of the allowed values of momenta determined by (64) would have been an eigenstate of the Hamiltonian. Though that is not the case, special linear combinations of the Bethe states do still solve the two magnon problem. It is however not clear to us if the above two body solutions can be utilized to solve the $n>2$ magnon problem. 


\section{Epilogue:}

The test of integrability carried out in the above analysis differs significantly from the usual one carried out to determine whether or not particular boundary conditions maintain the integrability of a given integrable spin chain. One usually declares a spin chain with open boundary conditions to be integrable if its bulk and boundary $S$ matrices satisfy the boundary Yang-Baxter or reflection algebra 33. This, for example, is the notion of integrability used in [4] for the open so(6) spin chain. However, it has to be kept in mind that the $S$ matrices satisfying the reflection algebra is a necessary and not a sufficient condition for integrability. Moreover, the bulk and boundary $S$ matrices truly become matrices when the impurities carry some internal degrees of freedom, which is not the case in the present problem, where the impurity can only be of one kind. The reflection algebras too, become non-trivial matrix relations only when the scattering matrices describe particles with internal degrees of freedom, and hence become non-commutative matrices. In a two component system like the present one, where the scattering matrices are just numbers depending on the momenta, the reflection equations are trivially satisfied. Thus, the analysis presented above also gives us a curious example of a situation where the necessary condition for integrability, namely the associated reflection algebra, is satisfied, while there does not appear to be a Bethe ansztz for the problem.

It is also instructive to compare the present study with those concerning the Inozemtsev spin chain. As mentioned before, the two (and three) loop su(2) dilatation operator in the sector of single trace operators has been shown to be embedded in a particular integrable long ranged spin chain known as the Inozemtsev spin chain. The Inozemtsev chain has a free parameter in which the Hamiltonian can be expanded and it is part of a complete set of commuting charges. It has been shown in 35] that it is possible to fine tune this free parameter so that up to the three loop order, the $s u(2)$ dilatation operator and the Inozemtsev spin chain become identical. Indeed, this is also the easiest way to establish perturbative integrability up to the three loop order in the $s u(2)$ sector of the gauge theory. However, the picture changes when one considers non-periodic boundary conditions. To the best of our knowledge, the only boundary conditions other than the periodic one that are compatible with the integrability of the Inozemntsev and other Inozemtsev like long ranged spin chains such as the celebrated Haldane Shastry model are special reflecting boundary conditions, where the spin chain interacts with its image on the other sides of the left and right boundaries in very specific ways, see for example [29, 28, 27]. The two loop (bulk) dilatation operator that we have in the present problem is the same one that arises in the analysis of the closed spin chains, and thus, it can be embedded in the Inozemtsev spin chain to this loop order. The boundary conditions in the present problem though are not of the reflecting type that lead to integrable 'open' versions of the Inozemtsev or Haldane Shastry 
spin chains which can possibly be an explanation for the absence of a consistent Bethe ansatz for the problem.

It is probably worth reiterating at this juncture that the two loop analysis presented in this paper is not the only example of the emergence of boundary conditions that lead to a potential violation of integrability of the dilatation operator of $\mathcal{N}=4 \mathrm{SYM}$. As mentioned earlier in the paper, the dynamical boundary conditions generated from the study of spin chains coupled to sub-determinant like operators in [5] produce quantum spin chains whose status, at least as far as integrability is concerned, is not clear. Another related scenario of a violation of integrability in this gauge theory arises when one studies the large $N$ limit of the $s o(6)$ invariant matrix model obtained as the dimensional reduction of the scalar sector of the super Yang-Mills theory to one (time) dimension. The quantization of the large $N$ limit of this matrix model was carried out recently by Klose in [47, and a breakdown of integrability of the model at higher loops was explicitly demonstrated.

As a final observation, it might be worth comparing the present analysis to the one performed in the context of defect conformal field theories. $\mathcal{N}=2$ defect conformal field theories are the conjectured gauge theory duals for the open string theory generated by wrapping a $D 5$ brane on an $A d S_{4} \times S^{2}$ subspace of $A d S_{5} \times S^{5}$. For the $s u(2)$ sector of such gauge theories, the open string degrees of freedom are generated by placing quark like fields at the ends of the spin chain, which result in open/free boundary conditions [36, 37, 38, 39, 40, 41, 42, 43, 44, 45, 46]. These are different from the boundary conditions that we encountered in the present work, but they are similar. For such open spin chains, an all loop Bethe ansatz was recently proposed in 34. Although, these are two different theories, and it probably does not make sense to compare their spectra, one can nevertheless compare the generic structure of the Bethe equation obtained in that analysis to the obstacles encountered in the present work. In the Bethe ansatz proposed in 34], the boundary scattering matrix did not have any dependence on the 't Hooft coupling of the gauge theory. Although the present analysis prevents us from writing down a set of Bethe equations of the present problem, we were able to determine the boundary scattering matrix at the two loop level, and found it to have a non-trivial dependence on the 't Hooft coupling. From the present analysis, it appears that such dependence is a generic feature of boundary scattering for (long-ranged) open spin chains, in the absence of reflective boundary conditions(see for example 28, 27]). Thus it might be a reasonable goal to perform an explicit two loop computation for the $s u(2)$ sector of $\mathcal{N}=2$ defect conformal field theories, as there might be hidden surprises to be uncovered. At the very least, it might be possible to understand their integrability by studying the relation between the boundary conditions that emerge out of the defect conformal field theories and the reflecting boundary conditions that have been studied in the context of long-ranged spin chains like the Haldane-Shastry and Inozemtsev chains in the past [29, 28, 27]. 
Thus in summary, the analysis presented in this paper presents some evidence in favor of lack of higher loop integrability in $\mathcal{N}=4 \mathrm{SYM}$ when one ventures into the study of the gauge theory dual of open string like operators. It is worth noting that similar obstacles in the direction of the implementation of Bethe ansatz methods at higher loops in the open string sector of this gauge theory were also arrived at recently in [48. The present analysis of integrability was carried out in a somewhat unconventional approach. It would perhaps be worthwhile to carry out a numerical study of the spectrum of the two loop spin chain presented in the paper along the lines of the involved work done in 10. The empirical knowledge of the spectrum can then be used to study whether or not the degeneracies responsible for the one loop integrability are lifted at two loops and possibly provide a more direct study of integrable aspects of the problem. A numerical investigation of the spectrum of the gauge thoery dual of open strings coupled to non-maximal giant gravitons was recently carried out in 49. In that paper some tell-tale numerical signs of integrability were also presented. Thus, it would be extremely interesting to understand if integrability is present in the problem in some way that is independent of the existence of a Bethe ansatz.

Acknowledgements: It is a great pleasure to thank Niklas Beisert, Dimitra Karabali, Tristan McLoughlin, Parameswaran Nair, Alexios Polychronakos, Sarada Rajeev, Radu Roiban and Matthias Staudacher for valuable discussions at various stages of this work. Special thanks are also due to Niklas Beisert, Tristan McLoughlin, Parameswaran Nair and Alexios Polychronakos for their comments on an earlier version of this manuscript.

\section{References}

[1] J. A. Minahan and K. Zarembo, JHEP 0303, 013 (2003) arXiv:hep-th/0212208.

[2] N. Beisert and M. Staudacher, Nucl. Phys. B 670, 439 (2003) arXiv:hep-th/0307042.

[3] N. Beisert, C. Kristjansen and M. Staudacher, Nucl. Phys. B 664, 131 (2003) arXiv:hep-th/0303060.

[4] D. Berenstein and S. E. Vazquez, JHEP 0506, 059 (2005) arXiv:hep-th/0501078.

[5] D. Berenstein, D. H. Correa and S. E. Vazquez, Phys. Rev. Lett. 95, 191601 (2005) arXiv:hep-th/0502172.

[6] N. Beisert and M. Staudacher, Nucl. Phys. B 727, 1 (2005) arXiv:hep-th/0504190.

[7] N. Beisert, C. Kristjansen, J. Plefka and M. Staudacher, Phys. Lett. B 558, 229 (2003) arXiv:hep-th/0212269. 
[8] M. Staudacher, JHEP 0505, 054 (2005) arXiv:hep-th/0412188.

[9] A. Rej, D. Serban and M. Staudacher, arXiv:hep-th/0512077.

[10] C. G. . Callan, J. Heckman, T. McLoughlin and I. J. Swanson, Nucl. Phys. B 701, 180 (2004) arXiv:hep-th/0407096.

[11] N. Beisert, Nucl. Phys. B 682, 487 (2004) arXiv:hep-th/0310252.

[12] A. Agarwal and G. Ferretti, JHEP 0510, 051 (2005) arXiv:hep-th/0508138.

[13] K. Peeters, J. Plefka and M. Zamaklar, JHEP 0411, 054 (2004) arXiv:hep-th/0410275.

[14] S. Bellucci and A. Marrani, arXiv:hep-th/0505106.

[15] V. Balasubramanian, M. Berkooz, A. Naqvi and M. J. Strassler, JHEP 0204, 034 (2002) arXiv:hep-th/0107119.

[16] S. Corley, A. Jevicki and S. Ramgoolam, Adv. Theor. Math. Phys. 5, 809 (2002) arXiv:hep-th/0111222.

[17] R. de Mello Koch and R. Gwyn, JHEP 0411, 081 (2004) arXiv:hep-th/0410236.

[18] E. Witten, JHEP 9807, 006 (1998) arXiv:hep-th/9805112.

[19] J. McGreevy, L. Susskind and N. Toumbas, JHEP 0006, 008 (2000) arXiv:hep-th/0003075.

[20] A. Hashimoto, S. Hirano and N. Itzhaki, JHEP 0008, 051 (2000) arXiv:hep-th/0008016.

[21] R. C. Myers, JHEP 9912, 022 (1999) arXiv:hep-th/9910053.

[22] M. T. Grisaru, R. C. Myers and O. Tafjord, JHEP 0008, 040 (2000) arXiv:hep-th/0008015.

[23] S. R. Das, A. Jevicki and S. D. Mathur, Phys. Rev. D 63, 024013 (2001) arXiv:hep-th/0009019.

[24] V. Balasubramanian, M. x. Huang, T. S. Levi and A. Naqvi, JHEP 0208, 037 (2002) arXiv:hep-th/0204196.

[25] D. Berenstein, Nucl. Phys. B 675, 179 (2003) arXiv:hep-th/0306090. 
[26] D. Serban and M. Staudacher, JHEP 0406, 001 (2004) arXiv:hep-th/0401057.

[27] D. Bernard, V. Pasquier and D. Serban, arXiv:hep-th/9501044.

[28] F. Finkel, D. Gomez-Ullate, A. Gonzalez-Lopez, M. A. Rodriguez and R. Zhdanov, Commun. Math. Phys. 233, 191 (2003) arXiv:hep-th/0202080.

[29] Xue. Wei-Xing and Wang. Yu-Peng Chinese. Phys. Lett 21, 537 (2004).

[30] A. Agarwal and S. G. Rajeev, Int. J. Mod. Phys. A 20, 5453 (2005) arXiv:hep-th/0409180.

[31] F. C. Alcaraz, M. N. Barber and M. T. Batchelor, PRINT-87-0215 (SAO-CARLOS)

[32] F. C. Alcaraz, M. N. Barber, M. T. Batchelor, R. J. Baxter and G. R. W. Quispel, J. Phys. A 20, 6397 (1987).

[33] E. K. Sklyanin, J. Phys. A 21, 2375 (1988).

[34] T. McLoughlin and I. J. Swanson, Nucl. Phys. B 723, 132 (2005) arXiv:hep-th/0504203.

[35] D. Serban and M. Staudacher, JHEP 0406, 001 (2004) arXiv:hep-th/0401057.

[36] O. DeWolfe and N. Mann, JHEP 0404, 035 (2004) arXiv:hep-th/0401041.

[37] B. Chen, X. J. Wang and Y. S. Wu, JHEP 0402, 029 (2004) arXiv:hep-th/0401016.

[38] B. Chen, X. J. Wang and Y. S. Wu, Phys. Lett. B 591, 170 (2004) arXiv:hep-th/0403004.

[39] D. Berenstein and S. A. Cherkis, Nucl. Phys. B 702, 49 (2004) arXiv:hep-th/0405215.

[40] K. Ideguchi, JHEP 0409, 008 (2004) arXiv:hep-th/0408014.

[41] Y. Susaki, Y. Takayama and K. Yoshida, Phys. Rev. D 71, 126006 (2005) arXiv:hep-th/0410139.

[42] Y. Susaki, Y. Takayama and K. Yoshida, Phys. Lett. B 624, 115 (2005) arXiv:hep-th/0504209.

[43] T. G. Erler and N. Mann, JHEP 0601, 131 (2006) arXiv:hep-th/0508064.

[44] N. Beisert and R. Roiban, JHEP 0511, 037 (2005) arXiv:hep-th/0510209. 
[45] K. Okamura, Y. Takayama and K. Yoshida, JHEP 0601, 112 (2006) arXiv:hep-th/0511139.

[46] D. Bundzik and T. Mansson, JHEP 0601, 116 (2006) arXiv:hep-th/0512093.

[47] T. Klose, JHEP 0510, 083 (2005) arXiv:hep-th/0507217.

[48] K. Okamura and K. Yoshida, arXiv:hep-th/0604100.

[49] D. Berenstein, D. H. Correa and S. E. Vazquez, arXiv:hep-th/0604123. 